\title{
Topological quantum catalyst: Dirac nodal line states and a potential electrocatalyst of hydrogen evolution in the TiSi family
}

\author{
Jiangxu $\mathrm{Li}^{1 \dagger}$, Hui Ma ${ }^{1,2 \dagger}$, Qing Xie ${ }^{1,3}$, Shaobo Feng ${ }^{1}$, Sami Ullah ${ }^{1,3}$, Ronghan $\mathrm{Li}^{1}$, Junhua Dong ${ }^{2}$, \\ Dianzhong $\mathrm{Li}^{1}$, Yiyi $\mathrm{Li}^{1}$ and Xing-Qiu Chen ${ }^{1 *}$
}

\begin{abstract}
Topological nodal line (DNL) semimetals, a closed loop of the inverted bands in its bulk phases, result in the almost flat drumhead-like non-trivial surface states (DNSSs) with an unusually high electronic density near the Fermi level. High catalytic active sites generally associated with high electronic densities around the Fermi level, high carrier mobility and a close-to-zero free energy of the adsorbed state of hydrogen $\left(\Delta G_{\mathrm{H}^{*}} \approx 0\right)$ are prerequisite to design alternative of precious platinum for catalyzing electrochemical hydrogen production from water. By combining these two aspects, it is natural to consider if the DNLs are a good candidate for the hydrogen evolution reaction (HER) or not because its DNSSs provide a robust platform to activate chemical reactions. Here, through first-principles calculations we reported a new DNL TiSi-type family, exhibiting a closed Dirac nodal line due to the linear band crossings in $k_{y}=0$ plane. The hydrogen adsorbed state on the surface yields $\Delta G_{\mathrm{H}^{*}}$ to be almost zero and the topological charge carries participate in HER. The results highlight a new routine to design topological quantum catalyst utilizing the topological DNL-induced surface bands as active sites, rather than edge sites-, vacancy-, dopant-, strain-, or heterostructure-created active sites.
\end{abstract}

Keywords: topological Dirac nodal line, semimetals, hydrogen evolution, catalyst

Topological semimetals [1], which have been classified into topological Dirac semimetals (TDs) [2-9], topological Weyl semimetals (TWs) [10-22], topological nodal line semimetals (DNLs) [23-38] and beyond [39], have been currently attracting extensively interest in con- densed matter physics and materials science. Different from TDs and TWs which exhibit isolated Dirac cones and Weyl nodes, DNLs [23-38] show a closed line near the Fermi level in their bulk phase. The projection of DNLs onto a certain surface would result in a closed ring in which the topologically quasi-flat drumhead-like nontrivial surface states (DNSSs) occur due to the non-trivial topological property of its bulk phase. This kind of exotic band structures exhibit various novel properties, such as giant surface Friedel oscillation in beryllium [27], flat Landau level [40] and long-range Coulomb interaction [41]. Recently, only DNL-induced DNSSs have been directly confirmed in beryllium [27] and DNLs have been, partially or indirectly, observed in several bulk materials, such as $\mathrm{PtSn}_{4}$ [42], $\mathrm{TlTaSe}_{2}$ [43], $\mathrm{PbTaSe}_{2}$ [44] and $\mathrm{ZrSiS}$ [45-47] as well as $\mathrm{Cu}_{2} \mathrm{Si}$ monolayer [48].

Topologically protected surface states in gold-covered $\mathrm{Bi}_{2} \mathrm{Se}_{3}$ topological insulators were theoretically suggested to serve as an effective electron path in the case of the $\mathrm{CO}$ oxidation [49]. Most recently, TWs (NbP, TaP, NbAs and TaAs) have been considered as excellent candidates of catalysts for hydrogen evolution reaction (HER) [50]. This key concept of TWs as catalysts was pioneered by topological surface states which provide an alternative way to create active sites, rather than by traditionally increasing the active edge sites or vacancies [51-60]. The possible bottleneck of TWs as electrocatalyst is their lower carrier density around Fermi level (Fig. 1a), because electrostatic screening strength in TWs is much weaker than that in the normal metals (e.g., Pt). DNL semimetal

\footnotetext{
${ }^{1}$ Shenyang National Laboratory for Materials Science, the Institute of Metal Research, Chinese Academy of Sciences, School of Materials Science and Engineering, University of Science and Technology of China, Shenyang 110016, China

${ }^{2}$ Environmental Corrosion Center, the Institute of Metal Research, Chinese Academy of Sciences, Shenyang 110016, China

${ }^{3}$ University of Chinese Academy of Sciences, Beijing 100049, China

† These authors contributed equally to this work.

* Corresponding author (email: xingqiu.chen@imr.ac.cn)
} 


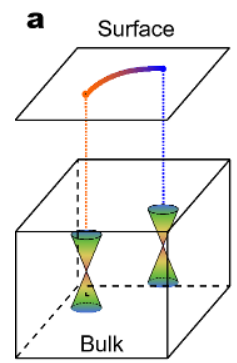

Weyl nodes

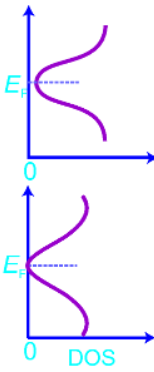

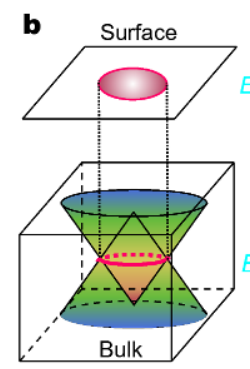

Dirac nodal line

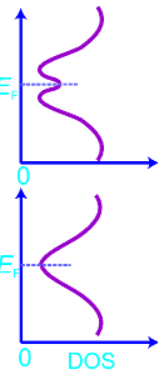

Figure 1 Schematics of momentum space diagrams and density of states (DOSs) of TWs and DNLs. (a) A pair of Weyl nodes in bulk (left lower panel) and broken Fermi arc surface states (left upper panel) on the surface and their corresponding DOSs (right panels); (b) a DNL in bulk (left lower panel) and the nearly flat drumhead-like non-trivial surface states on the surface (left upper panel) and their corresponding DOSs (right panels).

shows two distinguishing features from both TDs and TWs [50]. In bulk phase, a DNL results in a certain carrier density around Fermi level (Fig. 1b) and its DNSSs provide an unusually high electronic density around the Fermi level (Fig. 1b), as seen in metal beryllium [27]. Besides these advantages, similar to TDs and TWs, the DNL-induced surface states provide sufficient active planes (Fig. 1b) and the carrier mobility is, in principle, high, because the DNLs are formed by the continuous linear crossings of energy bands around Fermi level (Fig. 1b). Therefore, DNLs would be a better electrocatalyst for the HER due to three combined advantages: (i) topological DNL-induced DNSSs as robust active sites, (ii) high mobility and (iii) a certain carrier density around Fermi level. In addition, the crucial thermodynamic indicator as good electrocatalyst [51-64], the free energy of the adsorbed state $\left(\Delta G_{\mathrm{H}^{*}}\right)$, should be close to zero.

Within this context, through first-principles calculations (Methods, [62]) we report a new DNL family of the TiSi-type materials $M X(M=T i, Z r, H f ; X=S i, G e, S n)$. The DNL exists in $k_{y}=0$ plane of the bulk Brillouin zone (BZ) and induces the DNSSs, thereby resulting in a highly localized electronic density around Fermi level on the (010) surface. Interestingly, on the (010) surfaces of TiSi, the hydrogen adsorption free energies $\Delta G_{\mathrm{H}^{*}}$ are almost zero, much closer to zero than those values of all known catalysts for the HER [53-55, 61-64] including the most extensively used precious platinum $(\mathrm{Pt})$.

TiSi crystallizes in orthorhombic lattice (Fig. 2a) with the space group of Pnma (No. 62). The optimized lattice constants are $a=6.529 \AA, b=3.645 \AA$ and $c=5.004 \AA$, in good accordance with the previous experimental data [66-69] (Table S1). Si occupies the Wyckoff 4c site

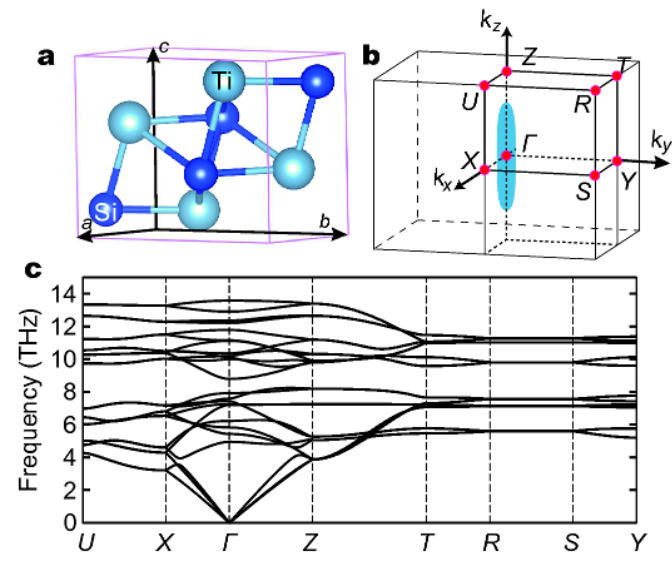

Figure 2 Crystal structure, Brillouin zone (BZ) and phonon dispersion of TiSi. (a) The orthorhombic lattice with the space group of Pnma, (b) the $\mathrm{BZ}$ and high symmetrical $k$-points of the lattice and the shaded region indicates the corresponding position of the Dirac nodal line at the $k_{y}=0$ plane, and (c) the DFT-derived phonon dispersion.

$(0.0362,0.2500,0.1103)$ and Ti is at another Wyckoff $4 c$ site $(0.1820,0.2500,0.6250)$. Additionally, the derived phonon dispersion does not show imaginary part of frequency and is dynamically stable (Fig. $2 b$ ).

To elucidate the unusual electronic properties of TiSi, we have compiled its electronic band structures in Fig. 3a, where the bands near the Fermi energy are without spinorbit coupling (SOC) inclusion. There are two quasilinear band crossing points, $\mathrm{A}$ and $\mathrm{B}$, as marked in Fig. 3a. Point $\mathrm{A}$ is located at $0.1 \mathrm{eV}$ above Fermi level in $X-\Gamma$ direction and $\mathrm{B}$ lies about $0.18 \mathrm{eV}$ below Fermi level along $\Gamma-Z$ direction, which are physically induced by the band inversion. At $\Gamma$ point, the center of BZ, the $d_{y z} \rightarrow d_{z^{2}}$ band inversion (Fig. S1 [65]) occurs between the two bands No. 1 and 2, as marked in Fig. 3a. Strikingly, these two bands not only cross at these two points, but also at a circle-like closed line around $\Gamma$ point in the $k_{y}=0$ plane (Fig. 3d), which is the apparent signature to DNL. The band crossing between No. 1 and 2 bands does not occur at the same energy level, but show a wave-like closed curve upon the $k$ vectors around $\Gamma$. This robust DNL stability is protected by the inversion and time-reversal symmetry without SOC effect. Because of light mass of $\mathrm{Ti}$ and $\mathrm{Si}$, their SOC effect is rather weak; therefore, it does not apparently affect the electronic band structures (Fig. S2) and the density of state (Fig. S3). Furthermore, the non-trivial topology order of TiSi is confirmed by the non-Abelian Berry connection method [71-73], as shown in Fig. $3 b, c$. In $k_{y}=0$ plane, the evolution loop of the Wannier center changes partners from $k_{x}=0$ to $\pi$. However, no partner changes in $k_{y}=\pi$ plane. Hence, the evo- 

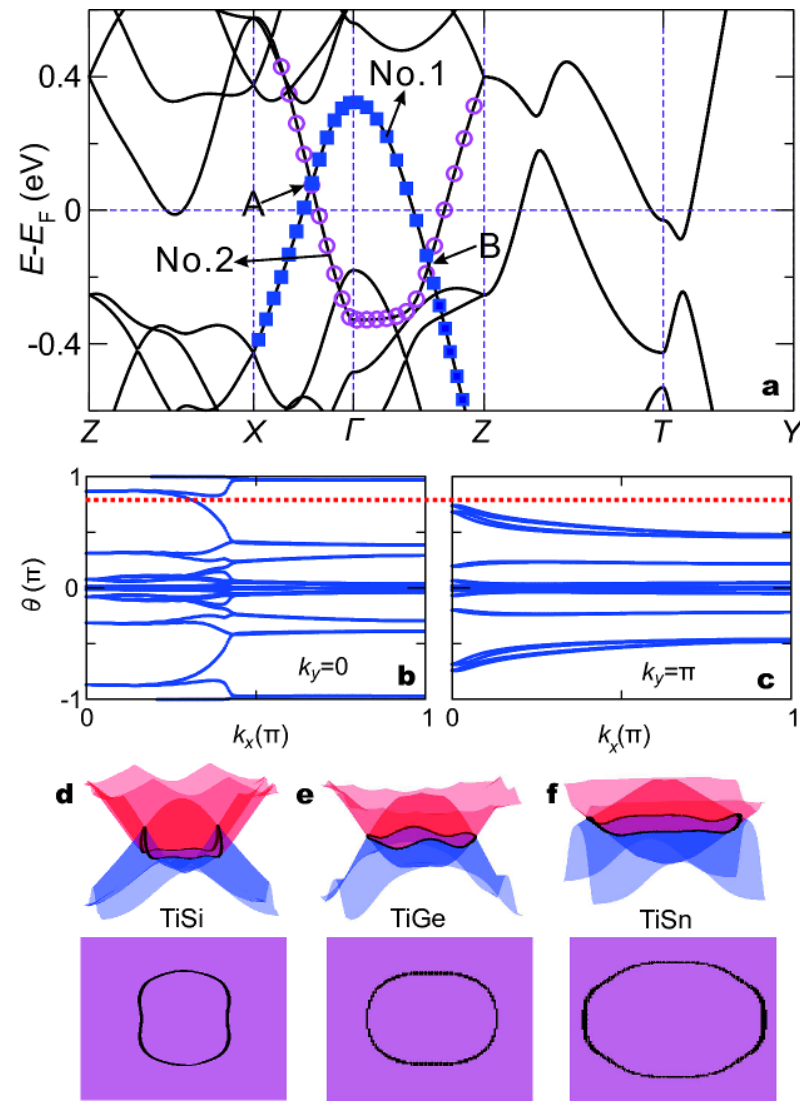

Figure 3 Electronic band structure and the evolution of Wannier centers of TiSi. (a) Calculated electronic band structure without the spinorbit coupling (SOC) inclusion. In (a), the hollow circles denote the weight of Ti $d_{y z}$-like states and the solid squares show the weight of the Ti $d_{z^{2}}$-like states, $(\mathrm{b}, \mathrm{c})$ the evolution of Wannier centers along the $k_{x}$ direction. The evolution lines cross the reference line (dotted red line) odd and even times in the $k_{y}=0$ and planes, respectively. $k_{y}$ and $k_{x}$ are in the directions, as given in Fig. $2 \mathrm{~b}$. (d-f) The Dirac nodal lines in the $k_{y}=0$ plane of TiSi, TiGe and TiSn, respectively. The upper and lower panels denote their three-dimensional visualizations and their corresponding two-dimensional projections on the (010) plane, respectively.

lution loop of the Wannier center cuts the reference line in odd times in $k_{y}=0$ plane, whereas it cross the reference line in even times in $k_{y}=\pi$ plane.

We have also considered the isoelectronic and isostructural TiGe, TiSn, HfSi, HfGe, HfSn, ZrSi, ZrGe and ZrSn. As shown in Table S1 and Figs. S1-S7 [65], TiGe (Fig. 3e) and TiSn (Fig. 3f) have similar electronic band structures to TiSi. However, due to heavy atomic mass with large SOC effect, the DNLs in the remaining compounds are broken into topological insulators.

We calculated the electronic structures of the (010) surface by varying the thickness of slabs (Fig. S8 [65]) to examine the topological surface bands for TiSi. As expected, the robust DNL-induced DNSSs (topological SF-

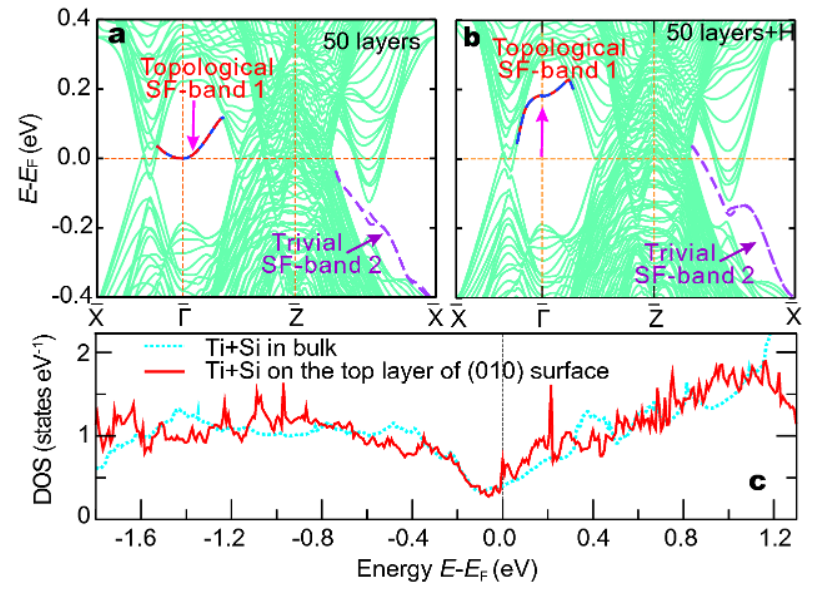

Figure $4(\mathrm{a}, \mathrm{b})$ Surface electronic structures on the 50-atomic-layer (010)-I surface without and with the hydrogen adsorption, respectively. The topologically protected DNSSs are marked as the SF-band 1 around $\Gamma$ and the trivial surface bands are marked as the SF-band 2 along $\Gamma$-X. (c) The comparison of the densities of states (DOSs) between the bulk phase and the top layer of the (010) surface of TiSi.

band 1 in Fig. 4a) appear when the slab's thickness is over eight atomic layers along the $b$-axis. From Fig. 4 a, outside the Dirac nodal ring, the two-fold degenerated topological SF-band 1 is clearly separated: one goes to the unoccupied conduction bands integrating with the projection of the electronic bands of bulk phase and the other one emerges into the valence bands overlapping with the projected bulk bands. In other words, these separated surface bands outside the projected Dirac nodal ring are topologically trivial and not correlated with the bulk DNL states. They mainly originate from the Ti $d_{x z}$, $d_{x y}$ and $d_{x^{2}-y^{2}-\text { like states, which means that the topological }}$ DNSSs (SF-band 1) only exist within the DNL-projected nodal ring on the (010) surface. The topologically protected SF-band 1 around $\Gamma$ point is mainly comprised of the $d_{y z}$ and $d_{z^{2}}$-like electronic states from the topmost atomic layer, reflecting well the $d_{y z} \rightarrow d_{z^{2}}$ band inversion in bulk phase (Fig. 3a). The SF-band 1 is two-fold degenerated and half-filled when the surface is electrically neutral, similar to the case of Be [27]. In addition, due to the DNSSs, the state density at Fermi level of the topmost atomic layer $(\mathrm{Ti}+\mathrm{Si})$ of the (010) surface is substantially larger by over $200 \%$ than that of its bulk phase (Fig. 4c). This fact can be reflected well by the DOS peak at the Fermi level at the surface, whereas the Fermi level is located at the deep pseudogap in bulk phase.

Importantly, these features of DNLs in TiSi motivate us to consider their electrocatalytic activity at (010) plane, as conceptually shown in Fig. 5a. In particular, the DNSSs (SF-band 1 in Fig. 4a) at (010) surface suggest the pos- 

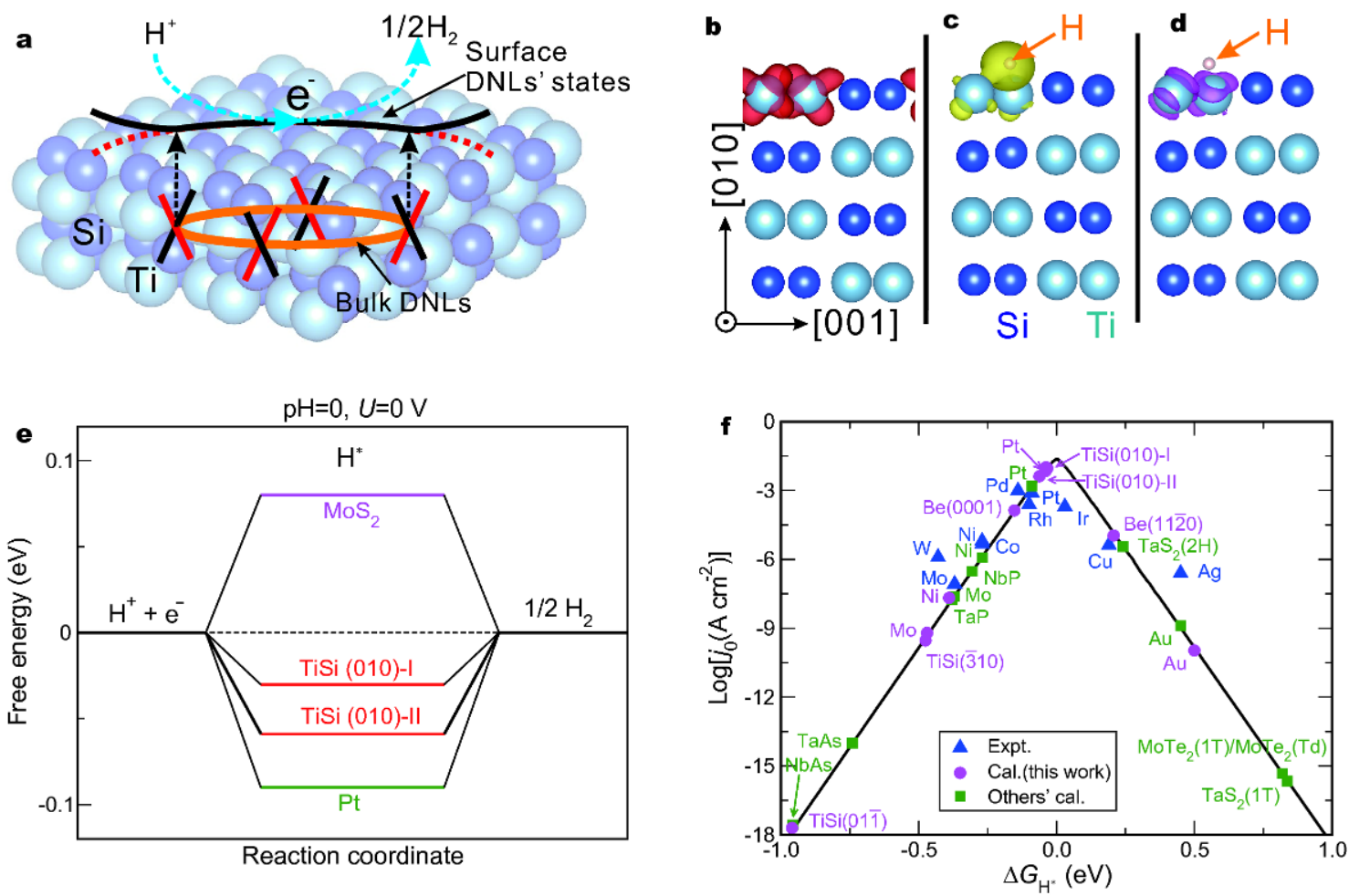

Figure 5 The effects of the topological DNLs on the HER activity of TiSi. (a) Schematic of the HER reaction of the DNL-induced DNSSs to provide active plane. (b) The visualized localized charges of the topological states on the (010)-I surface. The charges are characteristic of $d_{y z}$-like orbitals from the topmost Ti atoms. (c, d) The visualized localized charge accumulations of hydrogen and charge depletion surrounding Ti atom on the (010)-I surface, respectively. (e) Calculated free energy diagram for hydrogen evolution at a potential $U=0$ relative to the standard hydrogen electrode at $\mathrm{pH}$ $=0$. The free energy of $\mathrm{H}^{+}+\mathrm{e}^{-}$is by definition the same as that of $1 / 2 \mathrm{H}_{2}$ at standard condition of equilibrium (Methods [62]). The data of MoS $(-0.08 \mathrm{eV}$ for the edge states) and $\mathrm{Pt}(-0.09 \mathrm{eV})$ are taken from Ref. [52]. (f) Volcano plot for the HER of TiSi in comparison with various pure metals (the experimental data [61] of $\mathrm{Pt}, \mathrm{Pd}, \mathrm{Ni}, \mathrm{Ir}, \mathrm{Co}, \mathrm{Rh}, \mathrm{Ag}, \mathrm{Cu}, \mathrm{Mo}$ and $\mathrm{W}$ ), TWs (the calculated data [50] of $\mathrm{NbP}, \mathrm{TaP}, \mathrm{NbAs}$ and TaAs), and other candidates (the theoretical data [50] of $\mathrm{TaS}_{2}(2 \mathrm{H}), \mathrm{MoTe}_{2}\left(1 \mathrm{~T}^{\prime}\right), \mathrm{MoTe}_{2}(\mathrm{Td})$, and $\mathrm{TaS}_{2}(1 \mathrm{~T})$ ).

sibility of robust active planes for catalysis beyond defects, impurities and other surface modifications. Following the theoretical methods (see method [65] and as suggested in Refs. [51-64]) we evaluate the HER activity of (010), (01 $-1),(001)$ and $(-310)$ surfaces of TiSi. At each surface, the free energy $\left(\Delta G_{\mathrm{H}^{*}}\right)$ of the adsorbed state of hydrogen was determined by varying different adsorption sites and the slab thickness (Fig. S9 and Table S2 [65]). Furthermore, we have plotted the Volcano curves for comparing HER performance of TiSi with previous reports in Fig. $5 \mathrm{f}$. Remarkably, the (010) surface of TiSi exhibits a $\Delta G_{\mathrm{H}^{*}}$ closer to zero, at the top of the Volcano curve, than that of Pt $(-0.09 \mathrm{eV})$ [52], the edge states $(0.082 \mathrm{eV})$ [52] of $\mathrm{MoS}_{2}$ and a series of other noble metals as well as four known TWs (NbP, TaP, NbAs and TaAs) [50]. These facts demonstrate that the HER activity on the (010) surface of TiSi is highly attractive, attributed to its topologically protected DNSSs. The calculations reveal that, after the hydrogen adsorption on (010) surface, the topological SF-band 1 (Fig. 4b) becomes unoccupied above the Fermi level and, meanwhile, the hydrogen atom obtains the charges and is dispersed in deep energy region below Fermi level. This process can be made clearer by visualizing the localized charges in Fig. $5 \mathrm{~b}-\mathrm{d}$. On the clean (010) surface, the charges of the topological SFband 1 are localized at two nearest neighboring Ti atoms with the $d_{y z}$ orbitals (Fig. 5b). After the hydrogen adsorption, the topological charges are transferred to the hydrogen. As evidenced in Fig. 5c, a lone-pair s-like orbital appears as a result of the charge accumulations. The charge depletion of the two nearest neighboring Ti atoms are highly visualized in Fig. 5d, which also refers to the position of the localized topological charges on the $\mathrm{H}$-free adsorption (010) surface in Fig. 5b, indicating that the topological carriers on the SF-band 1 states are fully transferred into hydrogen $s$-like orbitals. 
In addition, according to the physics of the DNL, the DNL-induced DNSSs are mainly produced on the planes in parallel to DNLs. It is clear that very slight percentage of the DNSSs appear on the other $(-310)$ and $(01-1)$ planes rather than (001) or (100) planes because DNL is only located in the $k_{y}=0$ plane (Fig. 2b). Therefore, their HER activities on these surfaces would not be good expectedly. In fact, the $G_{\mathrm{H}^{*}}$ of $(-310),(01-1)$ and (001) planes are $-0.475 \mathrm{eV},-0.964 \mathrm{eV}$ and $-1.166 \mathrm{eV}$, respectively, far away from that of the (010) plane nearly at the top of the Volcano plot in Fig. 5f.

In summary, we have proposed the DNL semimetals as a promising alternative electrocatalyst for HER by the robust DNL-induced DNSSs as the active planes with a stable supply of itinerant electrons from the certain carrier density and the high mobility related with the DNLs. Furthermore, we have suggested the new DNL semimetal's family (TiSi, TiGe and TiSn) and theoretically demonstrate that their (010) surfaces, where the DNLinduced DNSSs exist, have a $\Delta G_{\mathrm{H}^{*}} \approx 0$ suitable for the excellent HER performance. This work paves the way for studying topological quantum catalyst using the concept of topological Dirac nodal line semimetals.

Received 8 December 2017; accepted 11 December 2017; published online 22 December 2017

1 Zahid Hasan M, Xu SY, Bian G. Topological insulators, topological superconductors and Weyl fermion semimetals: discoveries, perspectives and outlooks. Phys Scr, 2015, T164: 014001

2 Young SM, Zaheer S, Teo JCY, et al. Dirac semimetal in three dimensions. Phys Rev Lett, 2012, 108: 140405

3 Wang Z, Sun Y, Chen XQ, et al. Dirac semimetal and topological phase transitions in $\mathrm{A}_{3} \mathrm{Bi}(\mathrm{A}=\mathrm{Na}, \mathrm{K}, \mathrm{Rb})$. Phys Rev $\mathrm{B}, 2012,85$ : 195320

4 Liu ZK, Zhou B, Zhang Y, et al. Discovery of a three-dimensional topological Dirac semimetal, $\mathrm{Na}_{3} \mathrm{Bi}$. Science, 2014, 343: 864-867

5 Cheng X, Li R, Sun Y, et al. Ground-state phase in the threedimensional topological Dirac semimetal $\mathrm{Na}_{3} \mathrm{Bi}$. Phys Rev B, 2014, 89: 245201

6 Xu SY, Liu C, Kushwaha SK, et al. Observation of Fermi arc surface states in a topological metal. Science, 2015, 347: 294-298

7 Wang $\mathrm{Z}$, Weng $\mathrm{H}, \mathrm{Wu} \mathrm{Q}$, et al. Three-dimensional Dirac semimetal and quantum transport in $\mathrm{Cd}_{3} \mathrm{As}_{2}$. Phys Rev B, 2013, 88: 125427

8 Neupane M, Xu SY, Sankar R, et al. Observation of a three-dimensional topological Dirac semimetal phase in high-mobility $\mathrm{Cd}_{3} \mathrm{As}_{2}$. Nat Commun, 2014, 5: 3786

9 Liu ZK, Jiang J, Zhou B, et al. A stable three-dimensional topological Dirac semimetal $\mathrm{Cd}_{3} \mathrm{As}_{2}$. Nat Mater, 2014, 13: 677-681

10 Wan X, Turner AM, Vishwanath A, et al. Topological semimetal and Fermi-arc surface states in the electronic structure of pyrochlore iridates. Phys Rev B, 2011, 83: 205101

11 Weng H, Fang C, Fang Z, et al. Weyl semimetal phase in noncentrosymmetric transition-metal monophosphides. Phys Rev X, 2015, 5: 011029
12 Huang SM, Xu SY, Belopolski I, et al. A Weyl Fermion semimetal with surface Fermi arcs in the transition metal monopnictide TaAs class. Nat Commun, 2015, 6: 7373

13 Lv BQ, Weng HM, Fu BB, et al. Experimental discovery of Weyl semimetal TaAs. Phys Rev X, 2015, 5: 031013

14 Xu SY, Belopolski I, Alidoust N, et al. Discovery of a Weyl fermion semimetal and topological Fermi arcs. Science, 2015, 349: 613-617

15 Liu ZK, Yang LX, Sun Y, et al. Evolution of the Fermi surface of Weyl semimetals in the transition metal pnictide family. Nat Mater, 2015, 15: 27-31

16 Chang G, Xu SY, Sanchez DS, et al. A strongly robust type II Weyl fermion semimetal state in $\mathrm{Ta}_{3} \mathrm{~S}_{2}$. Sci Adv, 2016, 2: e1600295

17 Koepernik K, Kasinathan D, Efremov DV, et al. TaIrTe ${ }_{4}$ : a ternary type-II Weyl semimetal. Phys Rev B, 2016, 93: 201101

18 Soluyanov AA, Gresch D, Wang Z, et al. Type-II Weyl semimetals. Nature, 2015, 527: 495-498

19 Li FY, Luo X, Dai X, et al. Hybrid Weyl semimetal. Phys Rev B, 2016, 94: 121105

20 Ruan J, Jian SK, Zhang D, et al. Ideal Weyl semimetals in the chalcopyrites CuTlSe $, \mathrm{AgTlTe}_{2}, \mathrm{AuTlTe}_{2}$, and $\mathrm{ZnPbAs}_{2}$. Phys Rev Lett, 2016, 116: 226801

21 Ruan J, Jian SK, Yao H, et al. Symmetry-protected ideal Weyl semimetal in HgTe-class materials. Nat Commun, 2016, 7: 11136

22 Weng H, Fang C, Fang Z, et al. Coexistence of Weyl fermion and massless triply degenerate nodal points. Phys Rev B, 2016, 94: 165201

23 Fang C, Weng H, Dai X, et al. Topological nodal line semimetals. Chin Phys B, 2016, 25: 117106

24 Ryu S, Hatsugai Y. Topological origin of zero-energy edge states in particle-hole symmetric systems. Phys Rev Lett, 2002, 89: 077002

25 Heikkilä TT, Volovik GE. Dimensional crossover in topological matter: evolution of the multiple Dirac point in the layered system to the flat band on the surface. Jetp Lett, 2011, 93: 59-65

26 Burkov AA, Hook MD, Balents L. Topological nodal semimetals. Phys Rev B, 2011, 84: 235126

27 Li R, Ma H, Cheng X, et al. Dirac node lines in pure alkali earth metals. Phys Rev Lett, 2016, 117: 096401

28 Weng $\mathrm{H}$, Liang $\mathrm{Y}, \mathrm{Xu} \mathrm{Q}$, et al. Topological node-line semimetal in three-dimensional graphene networks. Phys Rev B, 2015, 92: 045108

29 Yu R, Weng H, Fang Z, et al. Topological node-line semimetal and Dirac semimetal state in antiperovskite $\mathrm{Cu}_{3} \mathrm{PdN}$. Phys Rev Lett, 2015, 115: 036807

30 Kim Y, Wieder BJ, Kane CL, et al. Dirac line nodes in inversionsymmetric crystals. Phys Rev Lett, 2015, 115: 036806

31 Xie LS, Schoop LM, Seibel EM, et al. A new form of $\mathrm{Ca}_{3} \mathrm{P}_{2}$ with a ring of Dirac nodes. APL Mater, 2015, 3: 083602

32 Zeng MG, Fang C, Chang GQ, et al. Topological semimetals and topological insulators in rare earth monopnictides. arXiv: $1504.03492 \mathrm{v} 1$

$33 \mathrm{Lu} \mathrm{L}, \mathrm{Fu} \mathrm{L}$, Joannopoulos JD, et al. Weyl points and line nodes in gyroid photonic crystals. Nat Photon, 2013, 7: 294-299

34 Mullen K, Uchoa B, Glatzhofer DT. Line of Dirac nodes in hyperhoneycomb lattices. Phys Rev Lett, 2015, 115: 026403

35 Gan LY, Wang R, Jin YJ, et al. Emergence of topological nodal loops in alkaline-earth hexaborides $\mathrm{XB}_{6}(\mathrm{X}=\mathrm{Ca}, \mathrm{Sr}$, and $\mathrm{Ba})$ under pressure. Phys Chem Chem Phys, 2017, 19: 8210-8215

36 Kawakami T, Hu X. Symmetry-guaranteed and accidental nodalline semimetals in FCC lattice. arXiv: 1611.07342v2

37 Yang B, Zhou H, Zhang X, et al. Dirac cones and highly aniso- 
tropic electronic structure of super-graphyne. Carbon, 2017, 113: 40-45

38 Chang G, Xu SY, Zheng $\mathrm{H}$, et al. Room-temperature magnetic topological Weyl fermion and nodal line semimetal states in halfmetallic Heusler $\mathrm{Co}_{2} \mathrm{TiX}$ (X=Si, Ge, or Sn). Sci Rep, 2016, 6: 38839

39 Bradlyn B, Cano J, Wang Z, et al. Beyond Dirac and Weyl fermions: unconventional quasiparticles in conventional crystals. Science, 2016, 353: aaf5037

40 Rhim JW, Kim YB. Landau level quantization and almost flat modes in three-dimensional semimetals with nodal ring spectra. Phys Rev B, 2015, 92: 045126

41 Huh Y, Moon EG, Kim YB. Long-range Coulomb interaction in nodal-ring semimetals. Phys Rev B, 2016, 93: 035138

42 Wu Y, Wang LL, Mun E, et al. Dirac node arcs in $\mathrm{PtSn}_{4}$. Nat Phys, 2016, 12: 667-671

43 Bian G, Chang TR, Zheng H, et al. Drumhead surface states and topological nodal-line fermions in $\mathrm{TlTaSe}_{2}$. Phys Rev B, 2016, 93: 121113

44 Bian G, Chang TR, Sankar R, et al. Topological nodal-line fermions in spin-orbit metal $\mathrm{PbTaSe}_{2}$. Nat Commun, 2016, 7: 10556

45 Schoop LM, Ali MN, Straßer C, et al. Dirac cone protected by nonsymmorphic symmetry and three-dimensional Dirac line node in ZrSiS. Nat Commun, 2016, 7: 11696

46 Neupane M, Belopolski I, Hosen MM, et al. Observation of topological nodal fermion semimetal phase in $\mathrm{ZrSiS}$. Phys Rev B, 2016, 93: 201104

$47 \mathrm{Hu}$ J, Tang Z, Liu J, et al. Evidence of topological nodal-line fermions in ZrSiSe and ZrSiTe. Phys Rev Lett, 2016, 117: 016602

48 Feng BJ, Fu BT, Kasamatsu S, et al. Experimental realization of two-dimensional Dirac nodal line fermions in monolayer $\mathrm{Cu}_{2} \mathrm{Si}$. Nat Commun, 2017, 8: 1007

49 Chen H, Zhu W, Xiao D, et al. CO oxidation facilitated by robust surface states on Au-covered topological insulators. Phys Rev Lett, 2011, 107: 056804

50 Rajamathi CR, Gupta U, Kumar N, et al. Weyl semimetals as hydrogen evolution catalysts. Adv Mater, 2017, 29: 1606202

51 Nørskov JK, Bligaard T, Logadottir A, et al. Trends in the exchange current for hydrogen evolution. J Electrochem Soc, 2005, 152: J23

52 Hinnemann B, Moses PG, Bonde J, et al. Biomimetic hydrogen evolution: $\mathrm{MoS}_{2}$ nanoparticles as catalyst for hydrogen evolution. J Am Chem Soc, 2005, 127: 5308-5309

53 Jaramillo TF, Jørgensen KP, Bonde J, et al. Identification of active edge sites for electrochemical $\mathrm{H}_{2}$ evolution from $\mathrm{MoS}_{2}$ nanocatalysts. Science, 2007, 317: 100-102

54 Nørskov JK, Bligaard T, Rossmeisl J, et al. Towards the computational design of solid catalysts. Nat Chem, 2009, 1: 37-46

55 Voiry D, Yamaguchi H, Li J, et al. Enhanced catalytic activity in strained chemically exfoliated $\mathrm{WS}_{2}$ nanosheets for hydrogen evolution. Nat Mater, 2013, 12: 850-855

56 Qu Y, Pan H, Kwok CT, et al. Effect of doping on hydrogen evolution reaction of vanadium disulfide monolayer. Nanoscale Res Lett, 2015, 10: 480

57 Greeley J, Nørskov JK, Kibler LA, et al. Hydrogen evolution over bimetallic systems: understanding the trends. ChemPhysChem, 2006, 7: 1032-1035

58 Zhang $\mathrm{F}$, Shi $\mathrm{Y}$, Xue $\mathrm{T}$, et al. In situ electrochemically converting $\mathrm{Fe}_{2} \mathrm{O}_{3}-\mathrm{Ni}(\mathrm{OH})_{2}$ to $\mathrm{NiFe}_{2} \mathrm{O}_{4}-\mathrm{NiOOH}$ : a highly efficient electrocatalyst towards water oxidation. Sci China Mater, 2017, 60: 324334

59 Zhang J, Chen M, Chen J, et al. Synthesis of single-crystal hyper- branched rhodium nanoplates with remarkable catalytic properties. Sci China Mater, 2017, 60: 685-696

60 Liu S, Zhang X, Zhang J, et al. $\mathrm{MoS}_{2}$ with tunable surface structure directed by thiophene adsorption toward HDS and HER. Sci China Mater, 2016, 59: 1051-1061

61 Greeley J, Jaramillo TF, Bonde J, et al. Computational highthroughput screening of electrocatalytic materials for hydrogen evolution. Nat Mater, 2006, 5: 909-913

62 Li H, Tsai C, Koh AL, et al. Activating and optimizing $\mathrm{MoS}_{2}$ basal planes for hydrogen evolution through the formation of strained sulphur vacancies. Nat Mater, 2016, 15: 48-53

63 Voiry D, Fullon R, Yang J, et al. The role of electronic coupling between substrate and $2 \mathrm{D} \mathrm{MoS}_{2}$ nanosheets in electrocatalytic production of hydrogen. Nat Mater, 2016, 15: 1003-1009

64 Conway BE, Tilak BV. Interfacial processes involving electrocatalytic evolution and oxidation of $\mathrm{H}_{2}$, and the role of chemisorbed H. Electrochim Acta, 2002, 47: 3571-3594

65 Supplemental Materials (1) computational details ([52,61,71-80]), (2) comparison of DFT optimized lattice constants with available experimental data, (3) band inversion in TiSi, (4) DNLs in TiSifamily, (5) Dirac nodal lines and Surface band structures in $\mathrm{ZrSi}$ and TiGe, (6) evolution of thickness-dependent surface electronic band structures of the TiSi (010) surface, and (7) computations of hydrogen adsorption on the TiSi surfaces

66 Agarwal S, Cotts EJ, Zarembo S, et al. The heat capacities of titanium silicides $\mathrm{Ti}_{5} \mathrm{Si}_{3}$, TiSi and $\mathrm{TiSi}_{2}$. J Alloys Compd, 2001, 314: 99102

67 Samsonov GV, Podgrushko NF, Dvorina LA. Thermal-conductivity of silicide phases of transition-metals of groups IV-VI. Inorg Mater, 1977, 13: 1429-1431

68 Samsonov GV, Okhremchuk LN, Podgrushko NF, et al. Relations between electron work function and certain physical-properties in silicides of group-IV transition-metals. Inorg Mater, 1976, 12: 720722

69 Brukl C, Nowotny H, Schob O, et al. Die Kristallstruckturen von TiSi, $\mathrm{Ti}(\mathrm{Al}, \mathrm{Si})_{2}$ und $\mathrm{Mo}(\mathrm{Al}, \mathrm{Si})_{2}$. Monatshefte für Chem, 1961, 92: 781-788

70 Fu L, Kane CL, Mele EJ. Topological insulators in three dimensions. Phys Rev Lett, 2007, 98: 106803

71 Soluyanov AA, Vanderbilt D. Computing topological invariants without inversion symmetry. Phys Rev B, 2011, 83: 235401

72 Yu R, Qi XL, Bernevig A, et al. Equivalent expression of $Z_{2}$ topological invariant for band insulators using the non-Abelian Berry connection. Phys Rev B, 2011, 84: 075119

73 Sun Y, Wang QZ, Wu SC, et al. Pressure-induced topological insulator in $\mathrm{NaBaBi}$ with right-handed surface spin texture. Phys Rev B, 2016, 93: 205303

Acknowledgements This work was supported by the National Science Fund for Distinguished Young Scholars (51725103), the National Natural Science Foundation of China (51671193 and 51474202), and the Science Challenging Project (TZ2016004). All calculations have been performed on the high-performance computational cluster in Shenyang National University Science and Technology Park and the National Supercomputing Center in Guangzhou (TH-2 system) with special program for applied research of the NSFC-Guangdong Joint Fund (the second phase) (U1501501).

Author contributions Chen XQ designed and supervised the project. Li J, Xie Q, Li R, Ullah S performed the DFT calculations of structural 
optimizations, electronic structures for all compounds. Li J performed the calculations of tight-binding modeling and surface states for DNLs. $\mathrm{Ma} \mathrm{H}$ and $\mathrm{Li} \mathrm{J}$ performed the calculations of hydrogen evolution reaction. Chen XQ analyzed results and wrote the paper with the inputs from Li J, Ma H, Ullah S, Li R. All authors including Li D, Dong J, Feng $\mathrm{S}$ and Li Y discussed the results and commented on the manuscript.
Conflict of interest The authors declare no conflict of interest.

Supplementary information The computational details, parameters, and any associated references are available in the supplementary material.
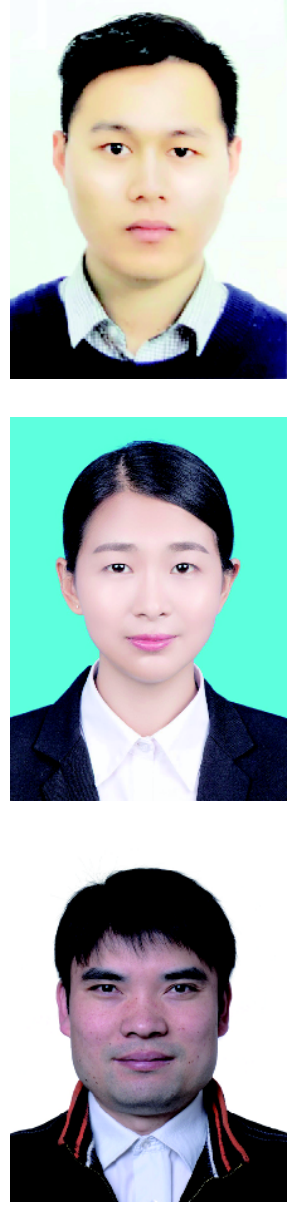

Jiangxu Li received his BSc degree from the Central South University in 2015. Now, he is a PhD candidate under the supervision of Prof. Xing-Qiu Chen at the Institute of Metal Research, Chinese Academy of Sciences, School of Materials Science and Engineering, University of Science and Technology of China. His research interest focuses on topological material design.

Hui Ma received her BSc degree from Hebei University of Technology in 2013. Now, she is a PhD candidate under the supervision of Prof. Junhua Dong and Prof. Xing-Qiu Chen at the Institute of Metal Research, Chinese Academy of Sciences, School of Materials Science and Engineering, University of Science and Technology of China. Her research interest focuses on electrochemical corrosion and catalysis.

Xing-Qiu Chen obtained his PhD degree in computational materials science at the University of Vienna in 2004 and completed his postdoctoral studies at Vienna Center of Computational Materials Science and Oak Ridge National Laboratory from 2005 to 2010. He is currently a professor at Shenyang National Laboratory for Materials Science, Institute of Metal Research, Chinese Academy of Sciences, funded by Hundred Talent Project and by the National Science Fund of Distinguished Young Scholars. His main scientific interests focus on the computational design of alloys including high-performance structural alloys, topological metals and alloys as well as the explorations of their potential applications.

\section{拓扑量子催化: TiSi家族的拓扑节线态和潜在催化析氢性能}

李江旭 ${ }^{1 \dagger}$, 马会 ${ }^{1,2 \dagger}$, 谢庆 ${ }^{1,3}$, 封少波 ${ }^{1}$, Sami Ullah ${ }^{1,3}$, 李荣汉 ${ }^{1}$, 董俊华 ${ }^{2}$, 李殿 ${ }^{1}$, 李依依 ${ }^{1}$ 陈星秋 ${ }^{1 *}$

摘要 拓扑节线半金属具有体能带反转形成的闭合环状狄拉克节线. 体狄拉克节线投影到某些表面会形成闭合的圈, 圈内会出现受拓扑 保护的能量色散非常小的鼓膜状表面态, 导致在费米能级具有高的电子密度. 目前, 为寻求贵金属铂的替代物, 新的析氢反应催化剂需要 具有高电子密度的催化活性位置, 高的载流子移动性和恰当的热力学稳定性 $(\Delta G \approx 0)$. 由鉴于此, 我们考虑具有稳定拓扑非平庸表面态的狄 拉克节线半金属是否可以作为析氢催化的平台. 基于第一性原理计算, 我们提出 $\mathrm{TiSi}$ 型拓扑节线态半金属新家族. 在其倒空间 $k_{y}=0$ 面, 反转 的两条能带相交形成闭合的狄拉克节线环. 氢吸附过程计算表明, 拓扑狄拉克节线完整投影的 $(010)$ 表面氢吸附自由能 $\Delta G$ 几乎为 0 . 并且, 拓扑电荷载流子也参与到了析氢过程. 综上, 我们提出拓扑狄拉克节线系统可以利用其鼓膜类拓扑表面态作为拓扑量子催化的平台, 并认 为 $\mathrm{TiSi}$ 家族材料有望成为析氢反应的催化剂, 这种催化设计路线主要利用拓扑节线材料非平庸鼓膜类表面态作为催化活性位置, 与以往传 统通过缺陷、界面修饰和构筑等调控催化剂存在本质不同. 